\title{
Performance Evaluation of H.264/AVC Intra Compound Image Compression System
}

\author{
R.Aparna \\ M.phil Research Scholar \\ Department of CS \\ Avinashilingam University
}

\author{
D.Maheswari \\ Ph.d Research Scholar \\ Department of CS \\ Avinashilingam University
}

\author{
Dr.V.Radha \\ Reader \\ Department of CS \\ Avinashilingam University
}

\begin{abstract}
This paper presents a comparison of H.264/AVC (Advanced Video Coding) based algorithms for compound images that contain text and natural images. Considered the problem of compressing compound images with a novel idea of using a single coder for compressing all the content types, we investigate the algorithm proposed by two models ZQ-CIC and D-CIC models. The coders presented in this study are very effective for compound documents because of its intraframe prediction mode. When using these coders, it is possible to improve significantly the quality of text regions, with little to negligible losses to the pictorial regions.
\end{abstract}

Keywords: H.264/AVC, D-CIC, ZQ-CIC

\section{INTRODUCTION}

The most important form of human communication today in "paper-less office" is paper documents. These documents are originated through computers and are stored in electronic form.

The obstacle faced while using electronic documents is that they can be quite large. Electronic document images often contain mixed content types like, text, background, graphics in both gray scale and in color form because of this mixed content, they are termed as "Compound Images"[1]. To deal effectively with such type of documents, it is necessary to detect layout and structural information from the image, and employ effective compression techniques that will suit the different content types of the image. Such techniques are termed as document image compression methods. Image compression considers minimization of storage space as its main objective. It is always desirable to have a coder that codes the images in such a way that it has the ability to process the resulting representation directly are desired.

In this paper two such coders for document image compression are explored and are analyzed in terms of its storage requirements (compression ratio) and processing flexibility (time). In order to see the advantages of each technique we compare different coding algorithms. The properties of two different compression algorithms are compared to JPEG 2000 Standard. Electronic compound images are represented in two forms namely, Vectorial and Raster images [2]. Vectorial images are normal images like natural pictures, which are not very much challenging since each object can be compressed individually and the whole file can be compressed losslessly. Compound documents are examples of raster images that contain a mix of text and pictorial contents. When compressing text, it is important to preserve the edges and shapes of characters accurately to facilitate reading. An example of Compound Image is shown in Fig. 1.

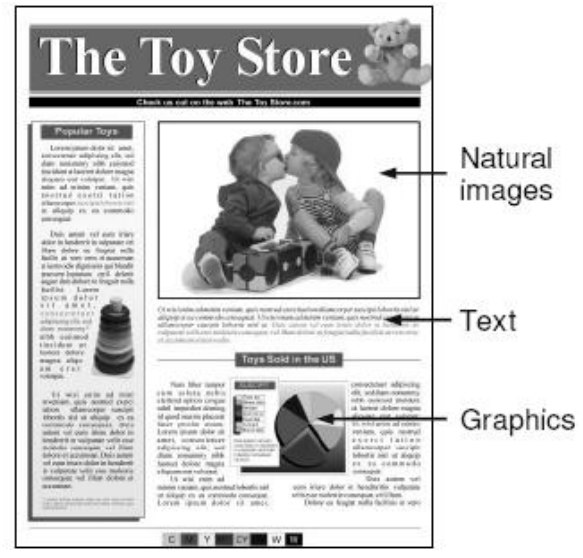

Fig.1: Example of Compound Image 
H.264 / Advanced Video Coding (AVC) is an industry standard for video compression [3]. The H.264 standard is also known as MPEG-4 Part 10 and is a successor to earlier standards such as MPEG-2 and MPEG-4. The H.264/AVC standard can deliver significantly improved compression efficiency compared with other standards. We use a single codec that will work efficiently for both compound and natural images. To satisfy this requirement we use D-CIC and ZQ-CIC Model that have proposed the use of H.264/AVC with Intra only compression for compound images.

\section{H.264/ADVANCED VIDEO CODING}

H.264 is an industry standard for video compression, the process of converting digital video into a format that takes up less capacity when it is stored or transmitted. Video compression (or video coding) is an essential technology for applications such as digital television, DVD-Video, mobile $\mathrm{TV}$, videoconferencing and Internet video streaming [4]. Here, an 'encoder' converts video into a compressed format and a 'decoder' converts compressed video back into an uncompressed format[5]. It defines a format (syntax) for compressed video and a method for decoding this syntax is used to produce a displayable video sequence. The Fig.2 shows the encoding and decoding processes and highlights the parts that are covered by the H.264 standard.

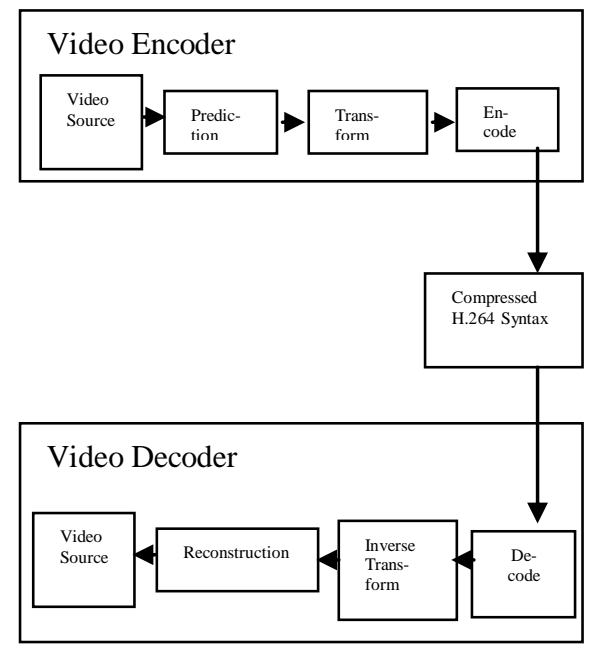

Fig.2: Encoding and Decoding Process

An H.264 video encoder carries out prediction, transform and encoding processes to produce a compressed H.264 bitstream. An H.264 video decoder carries out the complementary processes of decoding, inverse transform and reconstruction to produce a decoded video sequence. Compound images are compressed using two models D-CIC and ZQ-CIC[6,7]. The general steps followed for compressing the image by both the coders are:
- Color transformation from RGB image to YUV image

- $\quad$ Separate text and picture block

- Apply H.2.64/AVC coding algorithm to picture block

- Apply appropriate text block encoding techniques (either D-CIC model or ZQ-CID model)

- Display results

\subsection{D-CIC Model}

The D-CIC Model present an efficient compound image compression approach based on H.264/AVC intra coding. The input image in this model is divided into two regions, namely, text block and picture block[6]. A new coding scheme to extract and compress the text block is proposed. At a base, the H.264/AVC intra mode is used to compress the compound image. A spatial domain representation is chosen for text blocks and is described using base colors and an index map, which has the structure information of a text block.

\subsection{Zq-CIC Model}

H.264/AVC operating in intraframe mode is used to compress a compound image. Here, we take distortion into account separates the text and picture block. This approach used a segmentation-driven adaptation strategy to change the H.264/AVC quantization parameter on a macroblock by macroblock basis, i.e. bits are deviated from pictorial regions to text in order to keep text edges sharp. A region classification algorithm that will identify text and pictorial regions is applied. This classification algorithm is derived from an edge detector and identifies edges belonging to text as opposed to textures. Then each macroblock is classified into $16 \times 16$ pixels block. The binary image containing the segmented text is analyzed and each MB is classified as type 0,1 or 2 and a coding mask is constructed. Fig.3 is an example of Segmentation / Classification Algorithm:

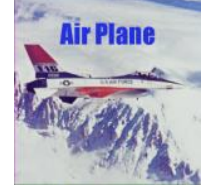

Original Image

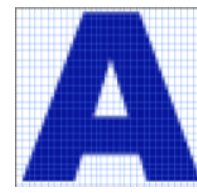

'A' in “Air"(Macroblock)
Fig.3: Segmentation / Classification Algorithm 


\section{IMPLEMENTATION}

In order to verify the performance of the two schemes we use variety of images for evaluation. This is performed using a series of different benchmark images and the characteristics of the images are selected in an assorted manner, that is, the images were a combination of pure picture, pure text and compound or mixed content type images. Both the models are also compared with the standard compressor JPEG 2000.To evaluate we use the following aspects:

\subsection{Compression Ratio}

This ratio measures the quantity of the compressed data in comparison to the quantity of the original data.

\section{Compression Ratio $=$ Length of Original Data}

\section{Length of Compressed Data}

From the above equation, it is obvious that as the compression ratio increases the compression technique employed is more effective.

Table 1: Compression Ratio

\begin{tabular}{|l|c|c|c|}
\hline \multicolumn{1}{|c|}{$\begin{array}{c}\text { Image } \\
\text { Name }\end{array}$} & D-CIC & ZQ-CIC & JPEG 2000 \\
\hline PI01 & 41.38 & 43.56 & 40.99 \\
\hline PI02 & 39.77 & 41.17 & 38.12 \\
\hline PI03 & 40.56 & 42.98 & 38.72 \\
\hline PT04 & 43.98 & 46.52 & 41.02 \\
\hline PIC_TEX01 & 35.56 & 38.05 & 34.56 \\
\hline PIC_TEX02 & 40.26 & 39.92 & 36.56 \\
\hline PIC_TEX03 & 34.52 & 37.05 & 33.92 \\
\hline MIXED & 43.46 & 46.08 & 41.69 \\
\hline
\end{tabular}

While comparing between the two models, ZQ-CIC produced better results than D-CIC.

\subsection{Compression and Decompression Time}

Compression and decompression time are the basic measurements used to evaluate an image compression system.

Compression and decompression time denotes the time taken for the algorithm to perform the encoding and decoding algorithms respectively.
Table 2: Compression Time

\begin{tabular}{|l|c|c|c|c|c|c|}
\hline \multirow{2}{*}{ Images } & \multicolumn{2}{|c|}{ D-CIC } & \multicolumn{2}{c|}{ ZQ-CIC } & \multicolumn{2}{c|}{ JPEG 2000 } \\
\cline { 2 - 7 } & CT & DT & CT & DT & CT & DT \\
\hline PI01 & 0.69 & 0.64 & 0.78 & 1.04 & 0.77 & 0.91 \\
\hline PI02 & 0.85 & 0.41 & 0.66 & 1.32 & 0.5 & 0.71 \\
\hline PI03 & 0.74 & 0.56 & 0.81 & 1.12 & 0.76 & 0.82 \\
\hline PT04 & 0.46 & 0.78 & 0.78 & 0.63 & 0.79 & 0.87 \\
\hline $\begin{array}{l}\text { PIC } \\
\text { TEX01 }\end{array}$ & 0.89 & 0.46 & 0.54 & 0.91 & 0.68 & 0.06 \\
\hline $\begin{array}{l}\text { PIC } \\
\text { TEX02 }\end{array}$ & 0.19 & 0.42 & 0.68 & 0.98 & 0.94 & 0.31 \\
\hline $\begin{array}{l}\text { PIC_ } \\
\text { TEX03 }\end{array}$ & 0.85 & 0.63 & 0.77 & 0.88 & 1.04 & 1.05 \\
\hline MIXED & 0.54 & 0.46 & 0.49 & 0.98 & 1.03 & 0.72 \\
\hline
\end{tabular}

The experimental results showed that we have a 30 per cent and 10 per cent positive gain in speed when H.264/AVC Intra coding is used for compressing compound images.

\subsection{Peak Signal to Noise Ratio (PSNR)}

PSNR is often used as a quality measurement between the original and a compressed image. The higher the PSNR, the better the quality of the compressed or reconstructed image. To compute the PSNR, the block first calculates the meansquared error using the following equation:

$\mathrm{MSE}=\frac{\sum_{\mathrm{M}, \mathrm{N}}\left[\mathrm{I}_{1}(\mathrm{~m}, \mathrm{n})-\mathrm{I}_{2}(\mathrm{~m}, \mathrm{n})\right]^{2}}{\mathrm{M}^{*} \mathrm{~N}}$

Then the block computes the PSNR for gray scale images using the following equation:

$$
\mathrm{PSNR}=10 \log 10\left[\frac{\mathrm{R}^{2}}{\mathrm{MSE}}\right]
$$

The PSNR for colour images with color components, R, G and $\mathrm{B}$ is:

$$
P S N R=10 \log _{10}\left[\frac{255^{2}}{\frac{\operatorname{MSE}(\mathrm{R})+\mathrm{MSE}(\mathrm{G})+\mathrm{MSE}(\mathrm{B})}{3}}\right]
$$


Table 3: PSNR

\begin{tabular}{|c|c|c|c|c|c|c|c|c|}
\hline \multirow{2}{*}{ Alg } & \multicolumn{3}{|c|}{ Pure picture } & \multirow{2}{*}{$\begin{array}{l}\text { Pure } \\
\text { text }\end{array}$} & \multicolumn{3}{|c|}{ Hybrid } & \multirow{2}{*}{$\begin{array}{c}\text { Compre } \\
\text { ssion }\end{array}$} \\
\hline & 01 & 02 & 03 & & 01 & 02 & 03 & \\
\hline $\begin{array}{l}\text { D- } \\
\text { CIC }\end{array}$ & $\begin{array}{c}42 . \\
36\end{array}$ & $\begin{array}{l}42 . \\
13\end{array}$ & $\begin{array}{l}42 . \\
12\end{array}$ & 46.02 & $\begin{array}{l}47 . \\
68\end{array}$ & $\begin{array}{l}46 . \\
86\end{array}$ & $\begin{array}{l}46 . \\
56\end{array}$ & 46.98 \\
\hline $\begin{array}{l}\text { ZQ- } \\
\text { CIC }\end{array}$ & $\begin{array}{l}42 . \\
87\end{array}$ & $\begin{array}{l}42 . \\
22\end{array}$ & $\begin{array}{l}42 . \\
14\end{array}$ & 45.02 & $\begin{array}{l}46 . \\
25\end{array}$ & $\begin{array}{l}45 \\
21\end{array}$ & $\begin{array}{l}44 . \\
32\end{array}$ & 45.18 \\
\hline $\begin{array}{l}\text { JPEG } \\
2000\end{array}$ & $\begin{array}{l}42 . \\
16\end{array}$ & $\begin{array}{l}42 . \\
01\end{array}$ & $\begin{array}{l}42 . \\
11\end{array}$ & 38.15 & $\begin{array}{l}41 . \\
68\end{array}$ & $\begin{array}{l}43 . \\
89\end{array}$ & $\begin{array}{l}45 . \\
02\end{array}$ & 42.55 \\
\hline
\end{tabular}

As it is evident, the D-CIC model produces better quality images than ZQ-CIC model for compound images.

\section{PERFORMANCE EVULATION}

The following Eight test images Fig 4. are the combination of all classes of compound images and were used for experiments:

The main goal of this test was to find which of the two algorithms is best suited for compound image compression. The system was compared with compression ratio, time and PSNR values. From the pattern obtained, it is evident that the PSNR and compression ratio are inversely related (i.e.) the PSNR decreases with the increase in compression ratio.

\section{CONCLUSION}

The results from the various experiments conducted showed that the both the models outperform the standard model with respect to compression ratio. While comparing between the two models, ZQ-CIC produced better results than D-CIC considering Pure Picture images. The D-CIC model produced better quality image after decompression when compared with ZQ-DIC model. As it is evident, the D-CIC model produces better quality images than ZQ-CIC model for compound images. From the results it could be concluded that applying H.264/AVC Intra coding to compound image compression can be considered as a success.

\section{REFERENCE}

[1]Jones, S. \& Marsh, S. (1996). Bi-level document image compression using layout information. (Working paper 96/01). Hamilton, New Zealand: University of Waikato, Department of Computer Science. [2]de Queiroz, R.L. (2005) Compressing Compound Documents, Document and Image Compression Handbook, edited by M. Barni, Marcel-Dekker.

[3]Milicevic, Z.M. and Bojkovic, Z.S. (2007) Intra/inter algorithm for B frame processing in H.264/AVC encoder, International Journal of Communications, Vol. 1, Issue 1, Pp.10-15.

[4]Richardson, I.E.G. (2009) Video compression design, analysis, consulting and research, Vcodex White Paper, http://www.vcodex.com/h264overview.html, Last Access Date : 09-07-2009.

[5]ISO/IEC JTC1/SC29 WG1, JPEG 2000 Committee, JPEG 2000 Verification Model (Technical Description), April 22, 1999.

[6] Ding, W., Lu, Y. and Wu,F. (2007) Enable Efficient Compound Image Compression in H.264/AVC Intra Coding, IEEE International Conference on Image Processing, Vol. 2, Pp. II - 337 - II - 340.

[7] Zaghetto, A. and de Queiroz, (2007) Using H.264/AVCIntra for Segmentation-Driven Compound Document Coding, IEEE ICIP 2007, Pp. II-333 - II-336.
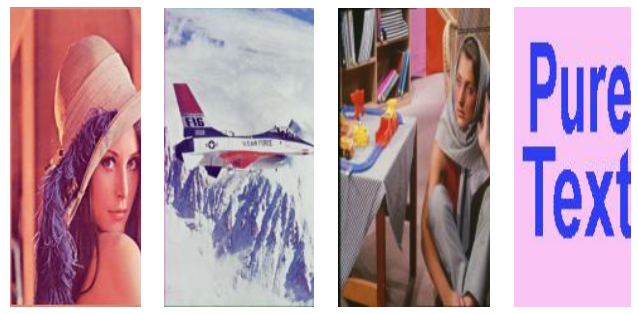

(a) PI01

(b) PI02

(c) PI03

(d)
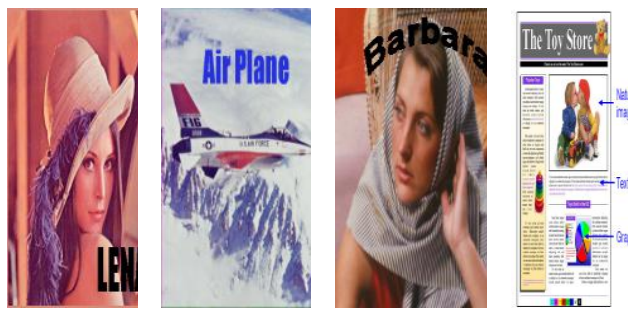

(e)
PIC_TE

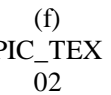

(g)

(h)

X01
PIC_TEX0

MIXE

Fig.4: Test Images 